\title{
A controlled trial of cyclophosphamide and azathioprine in Nigerian children with the nephrotic syndrome and poorly selective proteinuria
}

\author{
A. ADENIYI, R. G. HENDRICKSE, AND J. F. SOOTHILL \\ University College Hospital, Ibadan, Nigeria, and Institute of Child Health, London
}

SUMMARY In a controlled trial, symptomatic treatment alone, or 12 weeks of cyclophosphamide or azathioprine were compared in Nigerian children with nephrotic syndrome (mainly quartan malarial nephropathy) and poorly selective proteinuria. Full remission in 2 patients in each of the two groups treated with drugs, and diminution of proteinuria in most patients in the cyclophosphamide group showed possible evidence of benefit. Infections during treatment were significantly more common in the drug-treated groups but were controllable. Mortality from renal failure in the 2 nd year after treatment was significantly greater in the azathioprine-treated group than in the other two, suggesting that the drug may have exacerbated the nephritis. The 5-year survival rate was similar in the cyclophosphamide and the control group.

Nephrotic syndrome is common in Nigerian children and in most the disease is associated with Plasmodium malariae infection (Hendrickse and Gilles, 1963). Renal biopsies show an unusual type of glomerulonephritis (Hendrickse et al., 1972) which is associated with deposition of immunoglobulin, complement, and malarial antigen in the kidney (Houba et al., 1970; Hendrickse et al., 1972). Most such patients do not respond to corticosteroid drugs and may be made worse by them (Hendrickse et al., 1972). A corticosteroid-responsive minority is to be found among cases with less severe renal biopsy abnormality and highly selective proteinuria (Hendrickse et al., 1972; Adeniyi et al., 1976). No effective treatment is yet available for most patients and mortality is high.

Immunosuppressive treatment has been widely used in nephritis but controlled evidence of benefit is available only for corticosteroid-responsive nephrotic syndrome and SLE nephritis. Spontaneous recovery occurs in almost all kinds of nephritis. All the drugs used are toxic and increase susceptibility to infections; this is a serious problem in malnourished communities with poor sanitation.

University College of Ilorin, Nigeria

A. ADENIYI, professor of paediatrics

Alder Hey Children's Hospital, Liverpool

R. G. HENDRICKSE, professor of tropical paediatrics Institute of Child Health, London

J. F. SOOTHILL, professor of immunology
It has been suggested that susceptibility to nephritis may result from defective antigen elimination because of relative immunodeficiency (Soothill and Steward, 1971). Hence although immunosuppressive treatment may be valuable if it affects the damaging mechanism more than the elimination of antigen, it may make the nephritis worse if the reverse is true. We therefore assessed the effect on Nigerian nephrotic children with poorly selective proteinuria of a standard regimen alone, or with cyclophosphamide or azathioprine (randomly allocated), on proteinuria, survival, and incidence of complications.

\section{Patients and methods}

Children with nephrotic syndrome (oedema, heavy proteinuria, and serum albumin $<20 \mathrm{~g} / \mathrm{l})$ who presented sequentially at this hospital in Ibadan with nephrotic syndrome were divided into one of two groups according to differential protein clearance; those with highly selective proteinuria were included in a study of corticosteroid response (Adeniyi et al., 1976).

The present study was on 36 children ( 23 boys and 13 girls) aged between 2 and 12 years who had poorly selective proteinuria, clearance of IgG over clearance of albumin (CG/CA) $>15 \%$.

The patients were randomly allocated. Each drew a sealed card from a box, giving the name of one of 
the three groups-azathioprine, cyclophosphamide, or control. Cyclophosphamide was given orally as a single daily dose of $3 \mathrm{mg} / \mathrm{kg}$ per day for 12 weeks and azathioprine $2 \cdot 5-3 \mathrm{mg} / \mathrm{kg}$ per day similarly for 12 weeks. Haemoglobin, packed cell volume (PCV), white blood count (WBC), platelet counts, and differential protein clearance were done on all patients before starting treatment; a renal biopsy was done before treatment started in most of them.

Proteinuria was detected in urine by Albustix, and was graded $\mathrm{O}, \pm,+$ to $4+$ according to the manufacturer's scale. Differential protein clearance studies were performed on random samples of plasma and urine obtained at the first visit to hospital by the single radial gel diffusion technique (Adeniyi et al., 1976). Plasma albumin was standardised using a previously calibrated serum standard. PCV, WBC, and platelet counts were done 6 times in the first two weeks of treatment, and then at each visit. Renal biopsies were classified as previously described (Hendrickse et al., 1972).

Management. All patients entering the trial were in hospital for at least 2 weeks for diagnosis and starting treatment. They were then followed up at the outpatients department where they were seen every 2 weeks for 12 weeks, when cytotoxic treatment was stopped in most, after which they were seen less often for up to 5 years.

All patients were initially treated for malaria with a combination of chloroquine and primaquine, and received a high protein, low sodium diet, and the diuretic hydroflumethiazide. At each outpatient attendance drugs to last until the next visit were dispensed and any unused tablets were brought back to check on correct drug dosage at home. Patients were instructed to return immediately in the event of any untoward reactions or intercurrent illness. Full clinical examination, including blood pressure, was done at each visit and any illness was promptly treated, with hospital admission if necessary.

\section{Results}

There was no significant difference in ages, plasma albumin levels, CG/CA, or renal biopsies in the three groups. $P$. malariae were seen in blood films from 27 of the 36 patients. Blood urea and serum electrolytes were within normal ranges and only one patient had a blood pressure $>120 / 80 \mathrm{mmHg}$ at the first visit. 31 patients had renal biopsies; in all the lesions were characteristic of quartan malaria nephropathy. Immunofluorescence examination was positive for IgG in all 27 studied, and in all but one for C3.

Two patients treated with cyclophosphamide and 2 treated with azathioprine had complete clinical remissions with loss of proteinuria (trace or 0 ) by the 8th week of treatment, but none in the control group. Of these the 3 in whom biopsy was done showed mild QMN lesions, positive for IgG and C3. All patients showed a rise in serum albumin which was probably due to better feeding. Six other patients treated with cyclophosphamide improved considerably, as shown by loss of oedema, rise in serum albumin to normal levels, and reduction in proteinuria (Albustix + on 3 consecutive urine testings), which reflects a substantial reduction of albumin clearance (Table 1). The other children had no reduction of proteinuria detectable by these tests (Albustix $3+4+$ ) and serum albumin remained well below $20 \mathrm{~g} / 1$. More patients in the cyclophosphamide-treated group improved than did those in either the control or the azathioprine group.

The cytotoxic drugs had a number of adverse effects. Some patients complained of anorexia, nausea, and vomiting when either drug was given in the morning, but this was overcome by giving it at night. It was necessary to withdraw cyclophosphamide in 2 patients, in one because of severe haemorrhagic cystitis and in the other (subsequently shown to have severe renal amyloidosis) because of rapid deterioration, severe skin infection, bronchopneumonia, and septicaemia. Both drug-treated groups had significantly more infections than the control group. The main infections were of the skin and bronchopneumonia (Table 2). The organisms most often isolated from skin lesions were Staphylococcus aureus. The patients responded to treatment with antibiotics to which organisms were sensitive.

Table 1 Treatment groups and outcome in the 36 patients

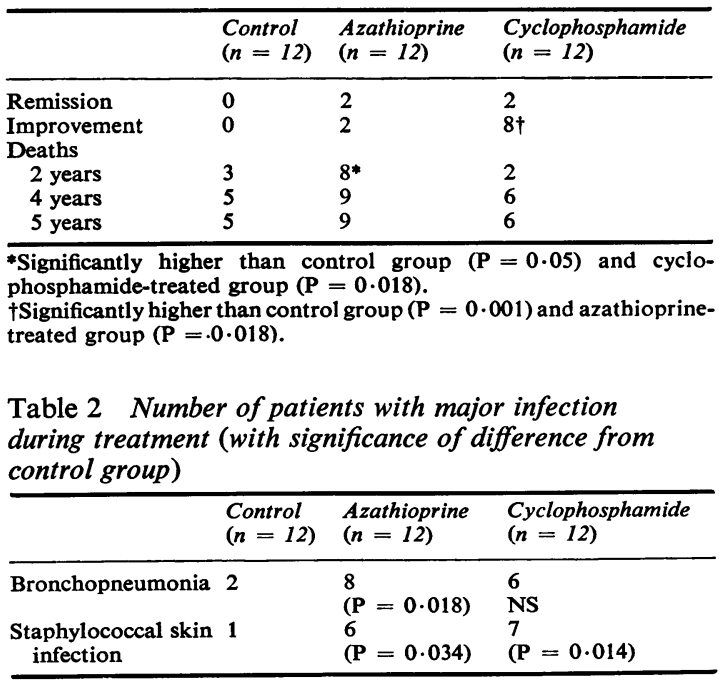


No patient died during the drug treatment period and in none did PCV, WBC, or platelet counts fall below normal limits.

Regular outpatient follow-up was planned for 5 years after the treatment. Some patients did not attend, and attempts were made to get into contact with them at home. The 5 patients who did not attend and were not traced, and who had had hypertension and raised levels of blood urea on the last visit, have been classified as presumed dead; one with normal blood pressure and blood urea after full remission at 4 years was presumed alive at 5 years. The 4 patients who had complete remissions remained well throughout follow-up. The mortality in the 5 years of followup is shown in the Figure. Only one child died in the first year (of renal failure due to renal amyloidosis). There were significantly more deaths in the azathioprine-treated group than in the other two, in the 2nd year of the follow-up (Table 1). There was no obvious relationship between time of death and the initial renal biopsy histology or differential protein clearance. In most children who died, death was preceded by hypertension and raised levels of blood urea and the deaths were regarded as being due to renal failure. At necropsy contracted kidneys were found in all.

The 4 patients who showed rapid disappearance of proteinuria remained well and free of proteinuria throughout the period of follow-up. The diminished proteinuria initially observed in the cyclophosphamide-treated group appears to have had no influence on prognosis as, after 2 years, mortality rose and was similar to that in the control group but was still lower than in the azathioprine-treated group.

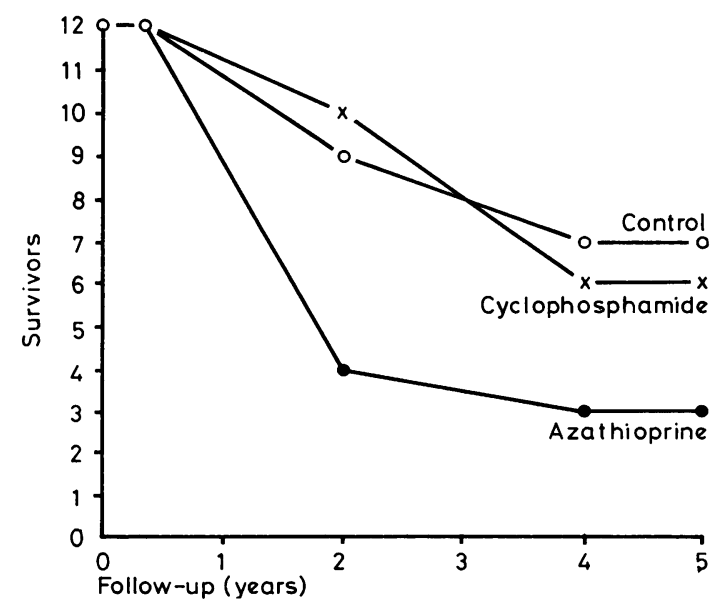

Figure Number of nephrotic children in each group surviving at each follow-up period. The allocation of nonattenders is explained in the text.
Since completing the trial cyclophosphamide has been used in 13 more such patients, and the same procedure was used. Three lost their proteinuria and 4 showed considerable reduction. Three other patients with nephrotic syndrome were given cyclophosphamide in gradually increasing doses until neutropenia (WBC $<3000 / \mathrm{mm}^{3} ;<3.0 \times 10^{9} / 1$ ) resulted when the drug was discontinued. No therapeutic benefit was noted. In 9 other patients treated with azathioprine no benefit of treatment was noted.

\section{Discussion}

The findings in this study confirm previous reports of the gravity of the prognosis in nephrotic syndrome in Nigerian children and the generally poor response to immunosuppressive treatment. Kibukamusoke (1968) reported an impression of benefit from treatment with azathioprine, not found in this or in previous studies undertaken in Ibadan (Adeniyi et al., 1970). Our findings show that azathioprine does not generally control proteinuria, and that its use is associated with a much higher risk of infection and a significant reduction in the 2-year survival rate. On the available evidence, azathioprine must be regarded as contraindicated in the treatment of nephrotic syndrome in West African children.

The increased mortality due to renal failure observed in patients treated with azathioprine is interesting and could be an example of the postulated system in which a drug may accelerate renal disease by causing more damage to mechanisms concerned with antigen clearance than to the mechanism causing the renal damage (Soothill and Steward, 1971; Steward et al., 1973). Some of the changes observed in immunofluorescence findings on renal biopsies in some azathioprine-treated cases of nephrotic syndrome (Houba et al., 1970) could be interpreted as having arisen from such an effect.

Cyclophosphamide treatment was associated with remission in 2 patients and a significant reduction of proteinuria in the group as a whole, but this was not associated with improved survival; there were serious side effects during treatment, especially infections. The absence of full remission in the control group and our previous experience, suggest that the occasional complete remission may be an effect of cyclophosphamide, and that response is more likely in those with a relatively short history ( $<3$ months), and relatively mild histological lesions (QMN grade I, Hendrickse et al., 1972). It seems sensible, therefore, to limit the use of this drug to patients who satisfy these criteria, and who can be closely supervised during treatment. Selectivity of proteinuria, useful for predicting corticosteroid response in 
Nigerian nephrotic children (Adeniyi et al., 1976) does not predict recovery after cyclophosphamide in those with relatively poorly selective proteinuria.

\section{References}

Adeniyi, A., Hendrickse, R. G., and Houba, V. (1970). Selectivity of proteinuria and response to prednisolone or immunosuppressive drugs in children with malarial nephrosis. Lancet, 1, 644-648.

Adeniyi, A., Hendrickse, R. G., and Soothill, J. F. (1976). Differential protein clearances and response to treatment in Nigerian nephrotic children. Archives of Disease in Childhood, 51, 691-696.

Hendrickse, R. G., and Gilles, M. (1963). The nephrotic syndrome and other renal diseases in western Nigeria. East African Medical Journal, 40, 186-201.

Hendrickse, R. G., Glasgow, E. F., Adeniyi A., White, R. H. R., Edington, G. M., and Houba, V. (1972). Quartan malarial nephrotic syndrome. Collaborative clinicopathological study in Nigerian children. Lancet, 1, 1143-1148.
Houba, V., Allison, A. C., Hendrickse, R. G., de Petris, S., Edington, G. M., and Adeniyi, A. (1970). Immune complexes in the nephrotic syndrome of Nigerian children. In Proceedings of the International Symposium on Immune Complex Diseases, Spoleto, June 1969, pp.23-35. Edited by L. Bonomo and J. L. Turk. Carlo Erba Foundation: Milan. Kibukamusoke, J. W. (1968). Malaria prophylaxis and immunosuppressant therapy in management of nephrotic syndrome associated with quartan malaria. Archives of Disease in Childhood, 43, 598-600.

Soothill, J. F., and Steward, M. W. (1971). The immunopathological significance of the heterogeneity of antibody affinity. Clinical and Experimental Immunology, 9, 193-199.

Steward, M. W., Alpers, J. H., and Soothill, J. F. (1973). The effect of cyclophosphamide on the antibody response. Proceedings of the Royal Society of Medicine, 66, 808-810.

Correspondence to Professor J. F. Soothill, Institute of Child Health, Department of Immunology, 30 Guilford Street, London WC1N 1EH.

Received 6 July 1978 